\title{
Evaluation of Fusion Based Biometric Recognition
}

\author{
Manju Dhanraj Pawar ${ }^{1}$ Dr.R. D. Kokate ${ }^{2}$ Dr.Vinodpuri Rampuri Gosavi ${ }^{3}$ \\ ${ }^{1}$ Research Scholar, G. S. Mandal's, M.I.T, Aurangabad Maharashtra, India \\ 2Principal , Government College of Engineering , Jalgaon Maharashtra, India \\ ${ }^{3}$ Assistant Professor, G. S. Mandal's, M.I.T, Aurangabad Maharashtra, India
}

\begin{abstract}
Article Info
Volume 7, Issue 6

Page Number: 323-333

Publication Issue :
\end{abstract}

November-December-2020

\section{Article History}

Accepted : 01 Dec 2020

Published : 10 Dec 2020

\section{ABSTRACT}

Now a days Biometric recognition plays a vital role in issues associated with identification off usurpation are currently at the heart of numerous concerns in our modern society. To work on connecting identity of an personality which is to be considered as an individual for various identification in the numerous operations of the state. Biometric authentication systems verify a person's claimed identity from behavioral traits like signature, voice or physiological traits like face, iris, fingerprint, palm. Biometric system of identification and confirmation provides automatic recognition of an individual based on certain unique features or characteristics possessed by that individual. It takes into consideration the natural features of every single individual for various applications in today's fast growing technical world. Biometric systems also help in overcoming the existing problems and limitations in authentication and identification fields and prove efficient and accurate in security related issues. In this paper we have focused and proposed a new era of fusion based recognition system.

Keywords : Fusion, Face Recognition, Iris, Speech, Sensitivity, Specificity and Accuracy

\section{INTRODUCTION}

Individual character alludes to an arrangement of characteristics like name, government managed savings number, signature and so forth that are connected with a man. Character administration is the procedure of making, keeping up and devastating personalities of people in a populace [1]. A solid character administration framework is earnestly required to battle the pandemic development in fraud and to meet the expanded security prerequisites in a mixed bag of utilizations going from global outskirt intersection to getting to individual data. Setting up (deciding or checking) the character of an individual is called individual acknowledgment or validation and it is a discriminating errand in any personality administration frame work. Biometric systems can be unimodal or multimodal. Unimodal biometric systems 
include only one biometric feature at a time for authentication or identification. Unimodal systems may give inaccurate results due to noise, or feature similarity to some extent. Multimodal systems include more than one biometric trait for identification. Multimodal biometric system overcomes the limitations of unimodal biometric systems such as non-universality, noise in sensed data, spoofing, intraclass variability, inter-class variability [3]. Multimodal biometric system can be constructed using more than one physiological or behavioral characteristic for identification and verification purposes. These types of systems are developed for security purposes in various fields like crime investigation, e-commerce and military purposes.

One of the problems with fingerprint recognition system is that fingerprint images have been observed to have poor ridge details. Similarly, face recognition system fails due to variation in facial expression. Hence while developing biometric systems the choice of biometric traits is important in order to achieve better performance. Multimodal systems available are face and ear [4], face and fingerprint, palm print and face, etc. In this proposed work, a unique trait iris is used to obtain a better performance and high security. figure 1 gives information of different biometric traits.

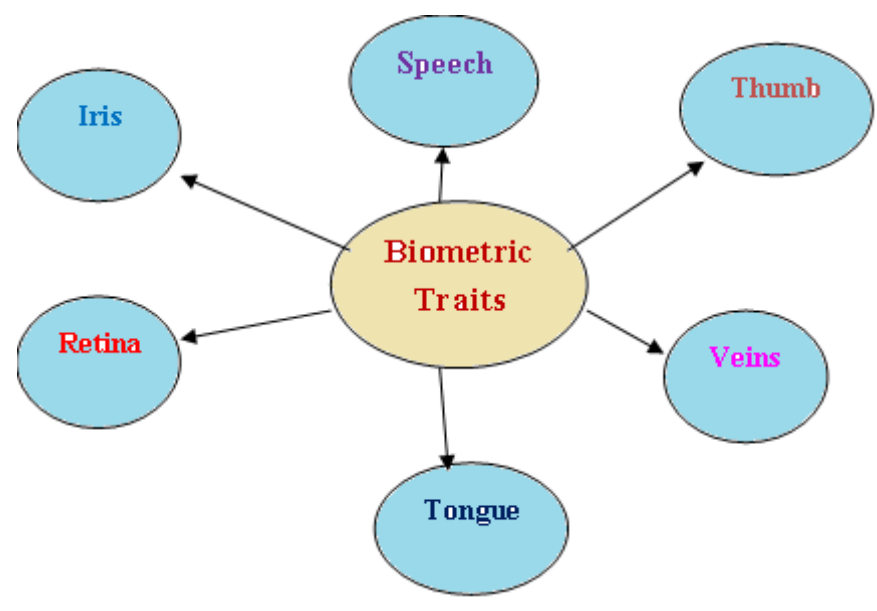

Figure 1 : Biometric Traits

\section{LITERATURE REVIEW}

There are several techniques proposed by different authors regarding various fusion based multimodal biometric systems. Multimodal biometrics has been proposed by Ross and Jain in 2003 . The concept of biometric multimodalities fusion is introduced with different fusion strategies and various levels of fusion are also presented [17, 19, 21 27-32]. Fusion of iris and fingerprint has attracted a lot of attention and researchers have presented variety of approaches in the literature [22, 23, 31, 33, 34].

Baig et al., in 2009 proposed a framework for multimodal biometric fusion based on utilization of a single matcher implementation for both modalities (iris and fingerprint). For their experiment they used the West Virginia University's multimodal database containing 400 images (4 enrolment images $\times 100$ users) and the threshold is set to the equal error rate EER. The comparison is being made in terms of percentage improvement in EER rather than the EER values themselves. Jagadeesan et al., in 2010 introduced a technique for cryptographic key generation by fusing fingerprint and iris biometrics. The fingerprint extractor is minutia based while the iris extractor is based on canny edge detector and Hough transform (Daugman's approach). The minutiae points and texture properties were first extracted from fingerprint and iris images, respectively, and then they were fused at the feature level to obtain the multi biometric template and subsequently a 256-bit secure cryptographic key from the multi biometric template is generated.

In 2011, Jameer Basha et al., introduced a new frame work for iris and fingerprint fusion at rank level; they conducted experimental tests using three implemented fusion methods: highest rank method, Borda count method, and logistic regression method. Their work achieved the best execution time required 
to match which is equal to 0.45 seconds for the highest rank method with optimal FAR and FRR equal, respectively to $0 \%$ and $0.25 \%$.

In 2012, Radha and Kavitha, presented a novel fusion scheme of fingerprint and iris modalities at feature extraction level. The scheme uses a concatenated feature vector from both iris and finger print. The log Gabor filter is used to extract the feature vectors of both modalities. then the phase data from 1D log Gabor filters is extracted and quantized to four levels to encode the unique pattern of iris and Fingerprint into bitwise biometric template. Hamming distance (HD) is used to generate a final match score. Experimental results were verified on database of 50 users accounting to $\mathrm{FAR}=0 \%$ and $\mathrm{FRR}=4.3 \%$.The execution time required to match is reduced to 0.14 seconds.

In 2013, Abdolahi et al., presented a multimodal biometric system (fingerprint and iris) using fuzzy logic and weighted code. After converting fingerprint and iris images to a binary code, the decision level fusion is used to combine the results. Fingerprint code is weighed as $20 \%$ and iris code as $80 \%$. The work achieved 2\% FAR and FRR and 98.3\% accuracy.

Sheetal Chaudhary, Rajender Nath et al., presented a vigorous multimodal biometric acknowledgment system participating iris, expression and fingerprint based on counterpart score level synthesis using multiple support vector machines. Here, MSVM are realistic in parallel manner to overcome the difficulty of absent biometric traits. It reflects every possible mixture of all the three biometric behaviours individually. They have used support vector machines as their approach and evaluated the recognition rate of 99 percent.
Divyakant T. Meva, C. K. Kumbharana et al., presented a biometric system which is getting popular since last years. As per the request of IT industry, this knowledge is satisfying verification and authorization requirements. But Unimodal Biometric schemes have their own limitations. To overcome the limitations of Unimodal Biometric Systems, they have choosen the method of Multimodal Biometric Schemes. In this paper, authors have assumed details about Multimodal Biometric system designed and developed to improve success ratio of verification. They have modified fingerprint and face acknowledgment approaches with match score based fusion. They have strained to identify achievement ration under numerous combinations of weights allocated to fingerprint and face counterpart scores. They have found the success rate of 93 percent with failure rates of 6.67 percent

Norsalina Hassan, Dzati Athiar Ramli, and Shahrel Azmin Suandi et al., proposed synthesis of face and fingerprint for vigorous recognition scheme. The addition is performed at the equivalent score level. The corresponding tasks for mutual modalities are approved out by using support vector machines. Trials on face expression and fingerprint record show that the presentations of multimodal biometric scheme provide better acknowledgment associated to unimodal biometric modality. They have used the sum rule and evaluated the error rate of 0.83 .

Yogesh. H. Dandawate, Sajeeda. R. Inamdar et al., shows seizing of three biometric characters of a person specifically face, pattern and palm vein by designed hardware well ahead these three behaviours pre-processed and fused collectively for cryptography. Palm is selected as a biometric attribute as no two palm veins counterpart unless they are of the similar person also palm has a decent vascular pattern creation as a good identifying issue for a person as likened to other biometric characters. They have used principle component analysis and gabor filtering and found the accuracy of $97 \%$ 
Nassima Kihal, Salim Chitroub and Jean Meunier et al., proposed a multimodal biometric scheme for verification, based on the synthesis of iris and palm print. They have proposed a method for feature abstraction of each modality using wavelet packet corrosion at four levels. This stretches 256 packets which can produce a compact binary cipher.

\section{Kamel Aizi Mohamed Ouslim Ahmed Sabri et al.,} presented a client-server network construction for a distant multimodal biometric credentials. As a substance of fact, they have used two modalities, the humanoid iris and his print impressions in order to reinforce the security, meanwhile the unimodal biometric schemes cannot always be castoff consistently to perform acknowledgment or authentications. They have used score level fusion and evaluated the false acceptance and false reject rates which were $24 \times 10^{-3}$ and $4 \times 10^{-4}$.

Poonam Sharma, Kulvinder Singh et al., proposed variety of glitches such as deafening data, various types of spoof attacks and intolerable error rate. These limits can be resolved by organizing multimodal biometric schemes. Multimodal biometric schemes exploit two or more separate traits, such as face, iris and fingerprint. Multimodal biometric schemes improve the acknowledgment accuracy more than single modal approaches. They have used multimodal biometric arrangements using fuzzy synthesis of fingerprint and face acknowledgment gives high accuracy as link to other fusion techniques. They have evaluated the accuracy of approximately $95 \%$.

Figure 2 gives the different level of fusion of multimodal biometric systems.

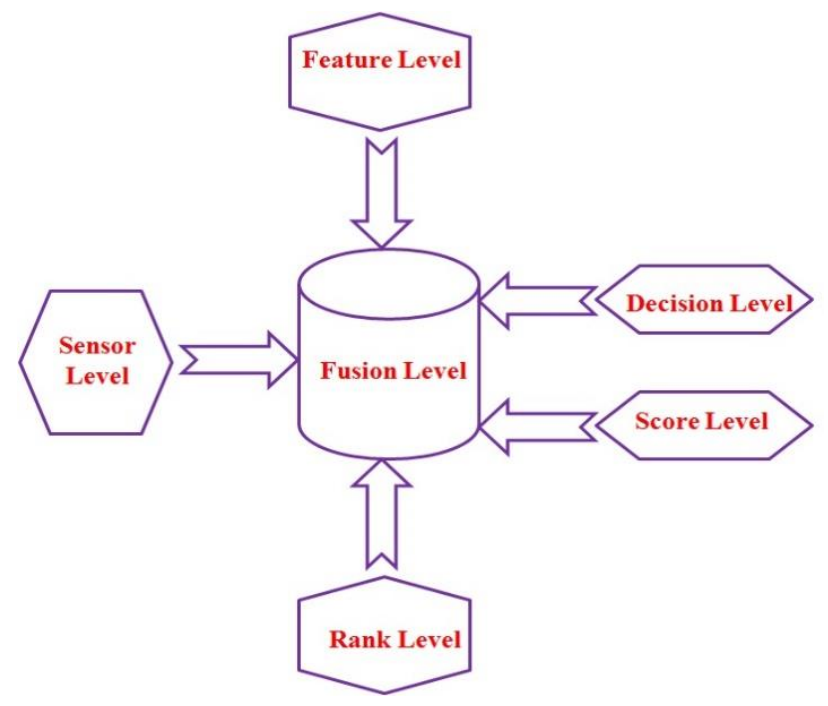

Figure 2 : Fusion Level in Multimodal Biometric Systems

\section{SYSTEM DEVELOPMENT}

The below is the proposed methodology diagram which will be used to achieve the above mentioned objectives in the efficient manner. The aim of our research is to develop and implement an effective algorithm for fusion based Biometric Recognition to get high efficiency rate of recognition. In this Performance Analysis we have experimented the proposed systems on various face database, Fingerprint database and evaluated the performance of systems in terms of efficiency, success criteria with different results.

Performance evaluation sections gives details about different evaluation metrics which we used for our experimentation and analysis. There are various performance matrics are being used for evaluation . 


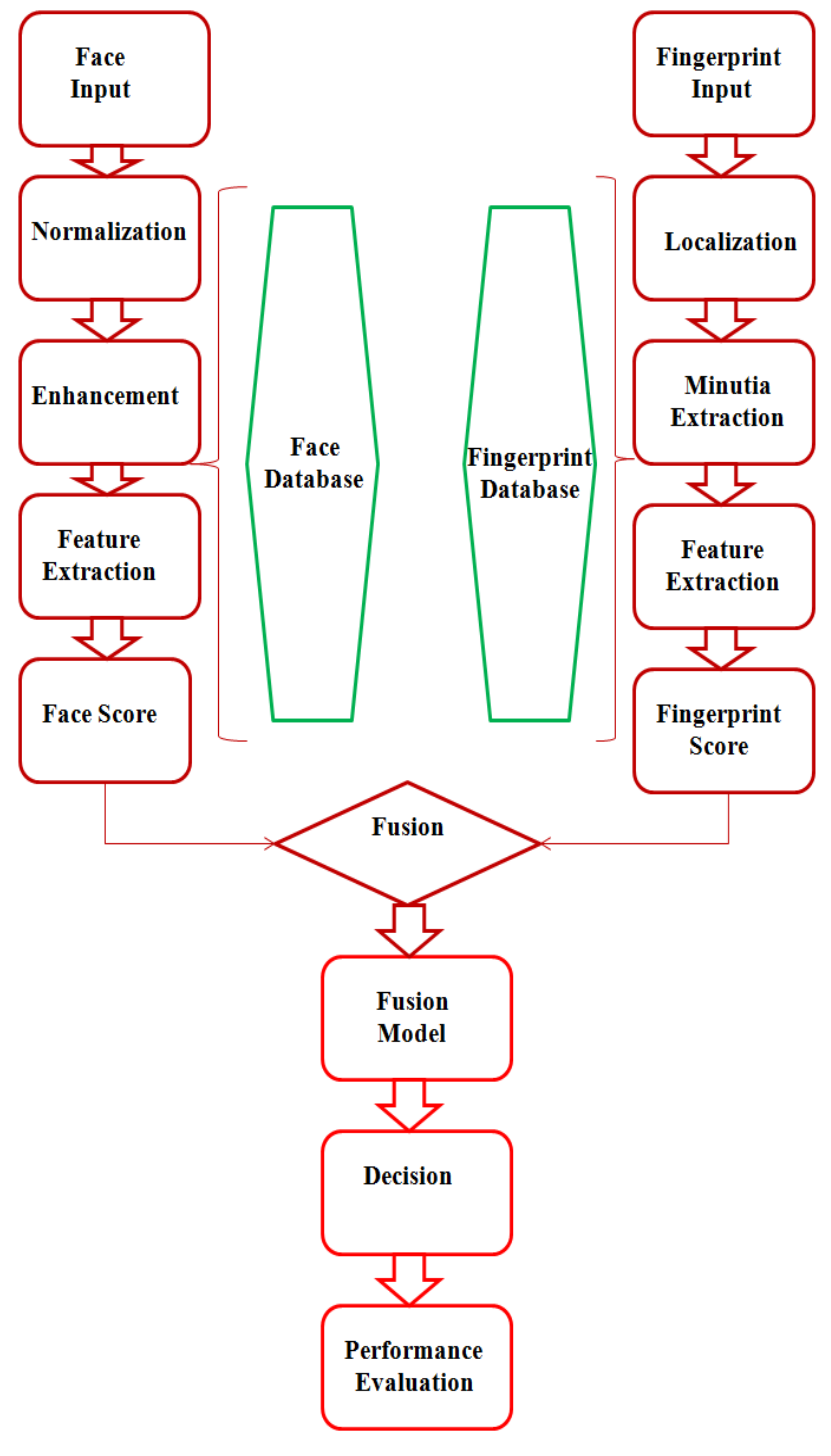

Figure 3 : System Development

The methodology is used to achieve our objective is Step 1: Firstly the User interface will be made in the MATLAB environment.

Step 2: Then the extractions for facial expressions will take place in which the features will be evaluated and extracted using principle component analysis.

Step 3: Then will apply feature optimization using firefly optimization for the facial category.

Step 4: The extraction of the minutiae will take place for the fingerprint category.

Step 5: The fusion process using summing rule will be evaluated
Step 6: Testing and Classification will be attained eventually.

Step 7: After full classifications for the multimodal biometric authentication the performance will be evaluated in terms of high sensitivity, high specificity and high recognition accuracy.

\section{PERFORMANCE EVALUATION}

This section deals with the efficient discussion of the simulated results in the MATLAB environment.

Various performance parameters are being used to test the systems like

False Acceptance Rate: The false acceptance rate (FAR) is the measure of the likelihood that, the biometric security system will incorrectly accept an access attempt by an unauthorized user. A system's FAR typically is stated as the ratio of the number of false acceptances divided by the number of identification attempts.

$\mathrm{FAR}=$

Totalno.ofillustrations-No.ofillustrationsFalselyaccepted Totalno.ofillustrations

$$
\operatorname{FAR}(\tau)=\operatorname{FMR}(\tau) *(1-\mathrm{FTA})
$$

False Rejection Rate : The false rejection rate, or FRR, is the measure of the likelihood that the biometric security system will incorrectly reject an access attempt by an authorized user. A system's FRR typically is stated as the ratio of the number of false recognitions divided by the number of identification attempts.

$\mathrm{FRR}=$

Totalno.ofillustrations-Noofillustrationsfalselyaccepted Noof illustrations

$$
\operatorname{FRR}(\tau)=\operatorname{FTA}+\operatorname{FNMR}(\tau) *(1-\text { FTA })
$$


Accuracy :The accuracy of a test is its ability to differentiate the patient and healthy cases correctly. Recognition accuracy is the ratio of number of faces correctly recognized upon number of faces used in testing Genuine and Impostor faces. To estimate the accuracy of a test, we should calculate the proportion of true positive and true negative in all evaluated cases. Mathematically, this can be stated as:

\section{Accuracy $=\mathrm{TP}+\mathrm{TN} / \mathrm{TP}+\mathrm{TN}+\mathrm{FP}+\mathrm{FN}$}

Where TP is True Positive, TN is True Negative, FP is False Positive and FN Is false negative. For the best recognition accuracy must be high .

Senstivity: Affectability measures the portion of positive cases that are named positive. It considers the proportion of positives that are correctly identified as such sensitivity.

$$
\text { Sensitivity }=\frac{\mathrm{TP}}{\mathrm{TP}+\mathrm{FN}}
$$

Specificity: Specificity measures the portion of negative cases that are named negative. Considers the proportion of negatives that are properly identified as such specificity.

$$
\text { Specificity }=\frac{T N}{F P+T N}
$$

Where, $\mathrm{TP}=$ true Positive, $\mathrm{TN}=$ True Negative, $\mathrm{FP}=$ False Positive and FN = False Negative. The model with the most outstanding specificity and precision is the best model.

Recognition Rate:It considers the section of number of faces recognized divided by number of faces presented.

$$
\mathrm{RR}=\frac{\text { number_of_faces_recognized }}{\text { number_of_faces_presented }}
$$

Receiver operating characteristic curve (ROC): plot of the rate of FMR as well as FAR (i.e., accepted impostor attempts) on the $\mathrm{x}$-axis against the corresponding rate of FNMR as well as FRR (i.e., rejected genuine attempts) on the $y$-axis plotted parametrically as a function of the decision threshold. An illustration of a ROC curve is presented in Graph
1 and 2 gives very important indicator to evaluate accuracy of the biometric systems.

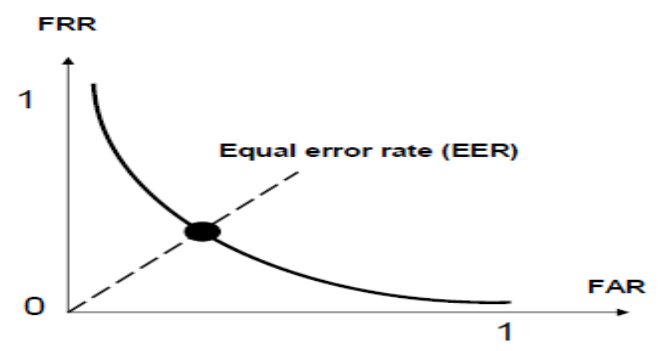

Graph 1 : An Illustration of ROC

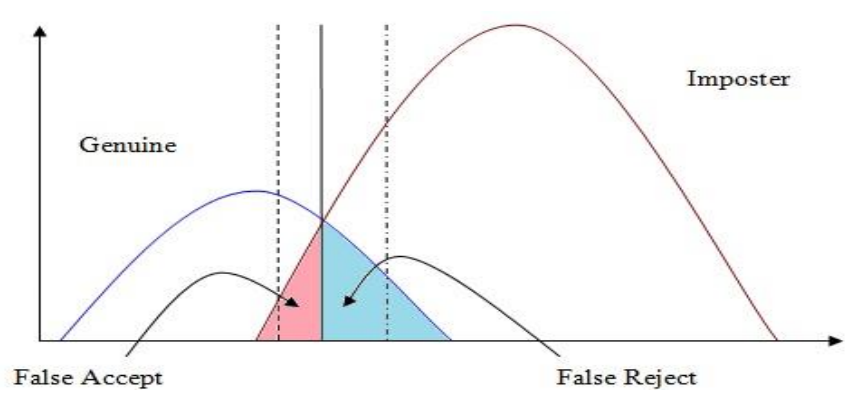

Graph 2 : FAR \& FRR

Table 1 shows the true positive rate which must be high for efficient multimodal biometric systems. For same specificity and sensitivity which must be high for the efficient authenticates For fusion based systems the recognition of the right individual and shows that the recognition is coming 0.92 in terms of probability and $92.03 \%$.

Table 1 : Performance Parameters

\begin{tabular}{|l|l|}
\hline Accuracy & 0.9285 \\
\hline Sensitivity & 0.9468 \\
\hline Specificity & 0.9263 \\
\hline False Negative & 0.0487 \\
\hline False Positive & 0.0743 \\
\hline True Negative & 0.9513 \\
\hline True Positive & 0.8657 \\
\hline
\end{tabular}

The FPR and FNR which is 0.07 and 0.04 which is measured very less and it must be low for the high 
stability and false detections of the individual samples.

The TPR and TNR which is 0.86 and 0.95 is always high to get the features of the test sample is closely matched with the training database for the high recognitions with less error rate probabilities

Graph 3 shows the performance parameters of FPR FNR and TPR TNR .

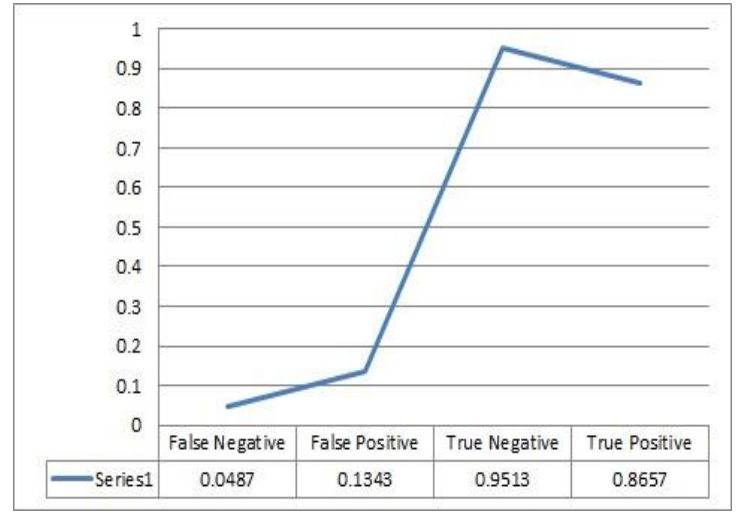

Graph 3 :FPR FNR and TPR TNR

Table 2 : System Test

\begin{tabular}{|r|c|c|}
\hline $\begin{array}{c}\text { Performance } \\
\text { Parameters }\end{array}$ & $\begin{array}{c}\text { Biometric } \\
\text { Recognition }\end{array}$ & $\begin{array}{c}\text { Not } \\
\text { Recognised }\end{array}$ \\
\hline Accuracy & 0.9201 & 0.92054 \\
\hline Sensitivity & 0.9168 & 0.91724 \\
\hline Specificity & 0.9235 & 0.92388 \\
\hline $\begin{array}{r}\text { False Positive } \\
\text { Rate (FPR) }\end{array}$ & 0.0759 & 0.075515 \\
\hline $\begin{array}{r}\text { False Negative } \\
\text { Rate (FNR) }\end{array}$ & 0.0839 & 0.083413 \\
\hline $\begin{array}{r}\text { True Positive } \\
\text { Rate (TPR) }\end{array}$ & 0.9241 & 0.92449 \\
\hline $\begin{array}{r}\text { True Negative } \\
\text { Rate(TNR) }\end{array}$ & 0.9161 & 0.91659 \\
\hline
\end{tabular}

The rank fusion based recognition rate equals (in \%): 92.01\%

Verification/authentication experiments:

The equal error rate equals : $5.03 \%$

The minimal half total error rate equals: $4.72 \%$
The verification rate at $1 \%$ FAR equals: $86.79 \%$ The verification rate at $0.1 \%$ FAR equals: $66.79 \%$ The verification rate at $0.01 \%$ FAR equals: $45.00 \%$

The performance comparison is to carried out for

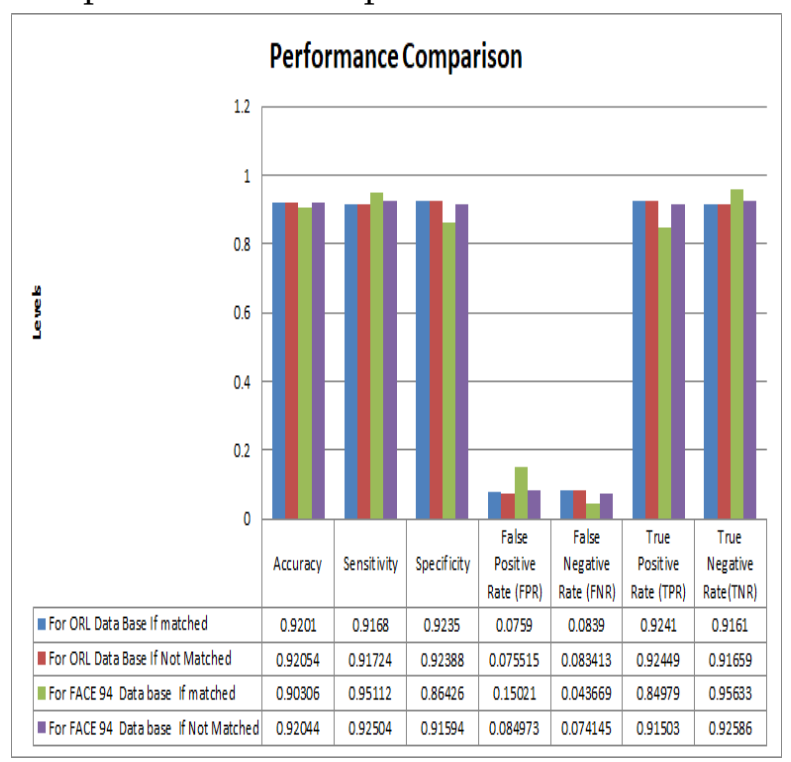

Graph 4 : Performance

The systems individual; being tested and got the results for same evaluation of systems as in table 3

Table 3: Individual Algorithm Test

\begin{tabular}{llllll}
\hline System & $\begin{array}{l}\text { Algorith } \\
\mathrm{m}\end{array}$ & $\begin{array}{l}\text { FA } \\
\mathrm{R}\end{array}$ & $\begin{array}{l}\text { FR } \\
\mathrm{R}\end{array}$ & $\begin{array}{l}\text { Efficien } \\
\text { cy }\end{array}$ & $\begin{array}{l}\text { Match } \\
\text { ed } \\
\text { Time }\end{array}$ \\
& & & & & $3.7 \mathrm{sec}$ \\
FACE & PCA & 0.6 & 9.8 & 92.85 & \\
& & 6 & 6 & & \\
Fingerpri & Gabor & 8 & 5 & 93.5 & $6.5 \mathrm{sec}$ \\
nt & HMM & & & &
\end{tabular}

After getting in to systems we have work on our systems and got developed fusion and had results as in table 4

Table 4 : Fusion Algorithm Test

\begin{tabular}{|c|c|c|c|c|c|}
\hline System & $\begin{array}{l}\text { Algorith } \\
\text { m }\end{array}$ & $\begin{array}{l}\text { FA } \\
\text { R }\end{array}$ & $\begin{array}{l}\text { FR } \\
\mathrm{R}\end{array}$ & $\begin{array}{l}\text { Efficien } \\
\text { cy }\end{array}$ & $\begin{array}{l}\text { Match } \\
\text { ed } \\
\text { Time }\end{array}$ \\
\hline FACE & $\mathrm{PCA}+\mathrm{AN}$ & 0.5 & 10.9 & 93.27 & $3.5 \mathrm{Sec}$ \\
\hline & FIS & 7 & 5 & & \\
\hline $\begin{array}{l}\text { Fingerpr } \\
\text { int }\end{array}$ & $\begin{array}{l}\text { Minutiae- } \\
\text { Hummin } \\
\text { g }\end{array}$ & 8 & 5 & 93.27 & 3.5 Sec \\
\hline
\end{tabular}


Finally all the fusion of face \& fingerprint has be done and traits are combined at matching score level using sum of scores technique. We got the the encouraging results of our systems and overall efficiency is improved up to $97.79 \%$ and these are very good results.

So graph 4 and 5 shows the False Acceptance Rate / False Rejection rate and EER with respect to considered threshold

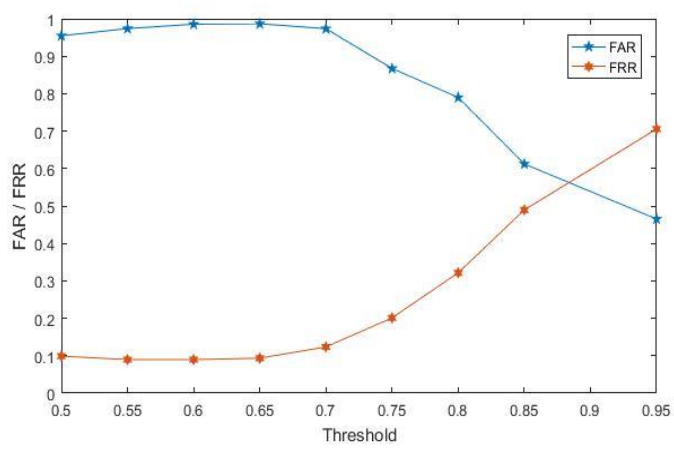

Graph 5 : FAR /FRR correlation w.r.t. threshold

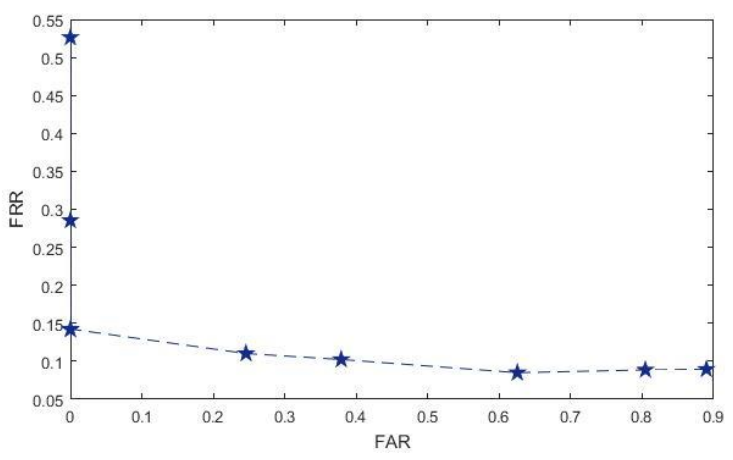

Graph 6 : EER of Fusion

\section{CONCLUSIONS}

In this paper, we carried out the system for fusion of face and fingerprint for recognition of individual identity of biometric multimodal systems. We have evaluated our systems on the performance parameters like Sensitivity, Selectivity, Specificity, FAR, FRR ,EER . We got improved results as compared to systems carried out by researchers as earlier. For evaluation and testing of our systems we have created our own database of 100 students from our organisation using various finger recognition sensors and cameras.

\section{REFERENCES}

[1]. Chlaoua, Rachid, Abdallah Meraoumia, Kamal Eddine Aiadi, and Maarouf Korichi. "Deep learning for finger-knuckle-print identification system based on PCANet and SVM classifier." Evolving Systems 10, no. 2 (2019): 261-272.

[2]. Hamd, Muthana H., and Marwa Y. Mohammed. "Multimodal Biometric System based Face-Iris Feature Level Fusion." Int. J. Mod. Educ. Comput. Sci 11, no. 5 (2019): 1-9.

[3]. Walia, G. S., Singh, T., Singh, K., \& Verma, N. (2019). Robust multimodal biometric system based on optimal score level fusion model. Expert Systems with Applications, 116, 364-376.

[4]. Dandawate, Yogesh H., and Sajeeda R. Inamdar. "Fusion based multimodal biometric cryptosystem." In Industrial Instrumentation and Control (ICIC), 2015 International Conference on, pp. 1484-1489. IEEE, 2015.

[5]. Kihal, Nassima, Salim Chitroub, and Jean Meunier. "Fusion of iris and palmprint for multimodal biometric authentication." In Image Processing Theory, Tools and Applications (IPTA), 2014 4th International Conference on, pp. 1-6. IEEE, 2014.

[6]. Aizi, Kamel, Mohamed Ouslim, and Ahmed Sabri. "Remote multimodal biometric identification based on the fusion of the iris and the fingerprint." In Electrical Engineering (ICEE), 2015 4th International Conference on, pp. 1-6. IEEE, 2015.

[7]. Shohel Sayeed, Ilham Nasir and Thian Song Ong, "An Efficient Multimodal Biometric Authentication Integrating Fingerprint and Face Features", American journal of applied 
sciences, science publications, pp. 1221-1227, 2016.

[8]. Jagadiswary, D., and D. Saraswady. "Biometric Authentication Using Fused Multimodal Biometric." Procedia Computer Science 85 (2016): 109-116.

[9]. Beritelli, Francesco, and Grazia Lo Sciuto. "Performance evaluation of multimodal biometric systems based on mathematical models and probabilistic neural networks." (2016).

[10]. Wild, Peter, Petru Radu, Lulu Chen, and James Ferryman. "Robust multimodal face and fingerprint fusion in the presence of spoofing attacks." Pattern Recognition 50 (2016): 17-25.

[11]. Assaad, Firas S., and Gursel Serpen. "Transformation based score fusion algorithm for multi-modal biometric user authentication through ensemble classification." Procedia Computer Science 61 (2015): 410-415.

[12]. Madane, Manisha, and Sudeep Thepade. "Score Level Fusion Based Bimodal Biometric Identification Using Thepade's Sorted n-ary Block Truncation Coding with Variod Proportions of Iris and Palmprint Traits." Procedia Computer Science 79 (2016): 466-473.

[13]. Jagadiswary, D., and D. Saraswady. "Biometric Authentication Using Fused Multimodal Biometric." Procedia Computer Science 85 (2016): 109-116.

[14]. Ammour, Basma, Larbi Boubchir, Toufik Bouden, and Messaoud Ramdani. "Face-Iris Multimodal Biometric Identification System." Electronics 9, no. 1 (2020): 85.

[15]. Vinodpuri Rampuri Gosavi, Dr. Anil Kishanrao Deshmane \& Dr. Ganesh Sahuba Sable, Adaptive Neuro Fuzzy Inference System for Facial Recognition, 10.9790/1676-1403011522, IOSR Journal of Electrical and Electronics Engineering (IOSR-JEEE) e-ISSN: 2278-1676,p-
ISSN: 2320-3331, Volume 14, Issue 3 Ser. I (May. - June. 2019), PP 15-22

[16]. R. Raghavendra, R. Ashok, and G. H. Kumar, "Multimodal biometric score fusion using gaussian mixture model and Monte Carlomethod," Journal of Computer Scienceand Technology, vol. 25, no. 4, pp. 771-782, 2010.

[17]. J. Zhou, G. Su, C. Jiang, Y. Deng, and C. Li, “A face and fingerprint identity authentication system based on multi-route detection," Neurocomputing, vol. 70, no. 4-6, pp. 922-931, 2007.

[18]. A. Ross and A. K. Jain, "multimodal biometrics: an overview," in Proceedings of the 12th European Signal Processing Conference (EUSIPCO '04), pp. 1221-1224, Vienna, Austria, September 2004.

[19]. H. Ailisto, E. Vildjiounaite, M. Lindholm, S.-M. M"akel"a, and J. Peltola, "Soft biometricscombining body weight and fat measurements with fingerprint biometrics," Pattern Recognition Letters, vol. 27, no. 5, pp. 325-334, 2006.

[20]. S. J. Xie, J. Yang, D. S. Park, S. Yoon, and J. Shin, "State of the art in biometrics," in Iris Biometric Cryptosystems, J. Yang and L. Nanni, Eds., InTech, 2011.

[21]. J. Yang and X. Zhang, "Feature-level fusion of fingerprint and finger-vein for personal identification," Pattern Recognition Letters, vol. 33, no. 5, pp. 623-628, 2012.

[22]. M. Abdolahi, M. Mohamadi, and M. Jafari, "Multimodal biometric system fusion using fingerprint and iris with fuzzy logic," International Journal of Soft Computing and Engineering, vol. 2, no. 6, pp. 504-510, 2013.

[23]. A. Baig, A. Bouridane, F. Kurugollu, and G. Qu, "Fingerprint - iris fusion based identification system using a single hamming distance matcher," International Journal of Bio-Science 
and Bio-Technology, vol. 1, no. 1, pp. 47-58, 2009.

[24]. R. N. Kankrale and S. D. Sapkal, “Template level concatenation of iris and fingerprint in multimodal biometric identification systems," International Journal of Electronics, Communication\& Soft Computing Science \& Engineering, pp. 29-36, 2012.

[25]. L. A. Zadeh, "Fuzzy sets," Information and Control, vol. 8, no. 3, pp. 338-353, 1965.

[26]. A. Ross and A. Jain, "Information fusion in biometrics," Pattern Recognition Letters, vol. 24, no. 13, pp. 2115-2125, 2003.

[27]. A. K. Jain and A. Ross, "Multibiometric systems," Communications of the ACM, vol. 47, no. 1, pp. 34-40, 2004.

[28]. D. R. Kisku, J. K. Sing,M. Tistarelli, and P.Gupta, "Multisensor biometric evidence fusion for person authentication using wavelet decomposition and monotonic-decreasing graph," in Proceedings of the 7th International Conference on Advances in Pattern Recognition (ICAPR '09), pp. 205-208, February 2009.

[29]. K. Sasidhar, V. L. Kakulapati, K. Ramakrishna, and K. K. Rao, "Multimodal biometric systems-study to improve accuracy and performance," International Journal of Computer Science and Engineering Survey, vol. 1, no. 2, pp. 54-60, 2010.

[30]. L. Lathaa and S. Thangasamy, "A robust person authentication system based on score level fusion of left and right irises and retinal features," Procedia Computer Science, vol. 2, pp. 111-120, 2010.

[31]. A. Jagadeesan, T. Thillaikkarasi, and K. Duraiswamy, "Cryptographic key generation from multiple biometric modalities: fusing minutiae with iris feature," International Journal of Computer Applications, vol. 2, no. 6, pp. 16-26, 2010.
[32]. H. F. Liau and D. Isa, "Feature selection for support vector machine-based face-iris multimodal biometric system," Expert Systems with Applications, vol. 38, no. 9, pp. 1110511111, 2011.

[33]. A. Jameer Basha, V. Palanisamy, and T. Purusothaman, "Efficient multimodal biometric authentication using fast fingerprint verification and enhanced iris features," Journal of Computer Science, vol. 7, no. 5, pp. 698-706, 2011.

[34]. N. Radha and A. Kavitha, "Rank level fusion using fingerprint and iris biometrics," Indian Journal of Computer Science and Engineering, vol. 2, no. 6, pp. 917-923, 2012.

[35]. U.Gawande, S. R.Nair,H. Balani, N. Pawar, and M. Kotpalliwar, "A high speed frequency based multimodal biometric system using iris and fingerprint," International Journal on Advanced Computer Engineering and Communication Technology, vol. 1, no. 2, pp. 66-73, 2012.

[36]. R. Giot and C. Rosenberger, "Genetic programming for multibiometrics," Expert Systems with Applications, vol. 39, no. 2, pp. 1837-1847, 2012.

[37]. J. Fierrez-Aguilar, J. Ortega-Garcia, and J. Gonzalez-Rodriguez, "Fusion strategies in multimodal biometric verification," in Proceedings of the IEEE International Conference on Multimedia and Expo (ICME '03), pp. 5-8, 2003. The Scientific World Journal 13

[38]. A. Jain, K. Nandakumar, and A. Ross, "Score normalization in multimodal biometric systems," Pattern Recognition, vol. 38,no. 12, pp. 2270-2285, 2005.

[39]. Chinese Academy of Sciences, Institute of Automation, "Database of 756 greyscale eye images," version 1.0, 2003, http://www .sinobiometrics.com.

[40]. “Database," 2013, http://www.idealtest.org 
[41]. D. Maltoni, R. Cappelli, J. L. Wayman, and A. $\mathrm{K}$. Jain, "FVC third fingerprint verification competition," in Proceeding of the International Conference on Biometric Authentication (ICBA04’ 04), pp. 1-7, Hong Kong, July 2004.

[42]. A. Abhyankar and S. Schuckers, "A novel biorthogonal wavelet network system for offangle iris recognition," Pattern Recognition, vol. 43, no. 3, pp. 987-1007, 2010.

[43]. L. Masek and P. Koveski, MATLAB Source Code for a Biometric Identification System Based on Iris Patterns, The University of Western Australia, 2003.

[44]. J. G. Daugman, "High confidence visual recognition of persons by a test of statistical independence," IEEE Transactions on Pattern Analysis and Machine Intelligence, vol. 15, no. 11, pp. 1148-1161, 1993.

[45]. A. K. Jain, L. Hong, S. Pankanti, and R. Bolle, "An identity authentication system using fingerprints," Proceedings of the IEEE, vol. 85, no. 9, pp. 1365-1388, 1997.

[46]. Houda Benaliouche andMohamed Touahria , "Comparative Study of Multimodal Biometric Recognition by Fusion of Iris and Fingerprint "Hindawi Publishing Corporation Scientific World Journal Volume 2014, Article ID 829369, 13 pages, http://dx.doi.org/ $10.1155 / 2014 / 829369$

\section{Cite this article as :}

Manju Dhanraj Pawar, Dr. R. D. Kokate Dr. Vinodpuri Rampuri Gosavi, "Evaluation of Fusion Based Biometric Recognition", International Journal of Scientific Research in Science and Technology (IJSRST), Online ISSN : 2395-602X, Print ISSN : 23956011, Volume 7 Issue 6, pp. 323-333, NovemberDecember 2020.

Available at doi :

https://doi.org/10.32628/IJSRST207648

Journal URL : http://ijsrst.com/IJSRST207648 